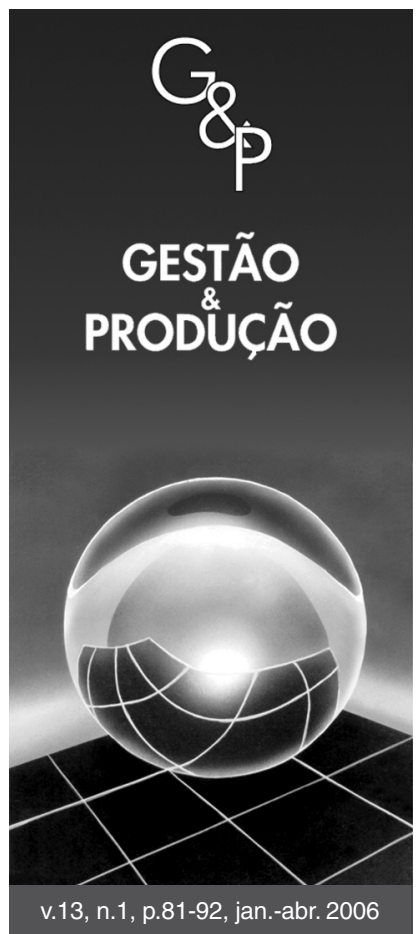

\title{
FATORES CRÍTICOS NA IMPLEMENTAÇÃO DO BALANCED SCORECARD
}

\author{
Vanderli Correia Prieto \\ Fábio Luis Alves Pereira \\ Marly Monteiro de Carvalho \\ Fernando José Barbin Laurindo \\ Escola Politécnica da USP, Av. Prof. Almeida Prado, 128, Tr.2 Biênio, \\ $2^{\circ}$ andar, CEP 05508-900, São Paulo, SP, Brasil, \\ e-mail: vanderli@prietoassociados.com.br, fabio.luis@ poli.usp.br, \\ marlymc@usp.br, fjblau@usp.br
}

Resumo

Recebido em 01/7/2004

Aceito em 27/11/2005

Existem diversas abordagens na literatura enfatizando a importância de promover o alinhamento estratégico e o seu desempenho operacional por meio do sistema de métricas. Um dos modelos mais difundidos é o balanced scorecard (BSC). A proposta deste artigo é discutir os fatores críticos de sucesso na implementação do BSC, bem como o processo de mudança e as técnicas envolvidas nos bastidores da implementação, tendo como objeto central o confronto entre a perspectiva da empresa de consultoria que implementa o BSC e da empresa que adota o método. A abordagem metodológica utilizada foi a de estudo de múltiplos casos. A pesquisa foi organizada baseada em um estudo de casos múltiplos criado sobre uma estrutura teórica (framework) extraído da literatura especializada e submetido a empresas de consultoria focadas neste tipo de implementação para identificar e listar estes aspectos críticos nas empresas brasileiras. Em uma segunda etapa da pesquisa, um estudo de caso foi realizado com o objetivo de analisar os mesmos aspectos sob o ponto de vista das empresas que adotam a metodologia, as empresas clientes.

Palavras-chave: balanced scorecard (BSC), alinhamento estratégico, implementação da estratégia, sistemas de indicadores de desempenho.

\section{Introdução}

A relevância entre o alinhamento da estratégia de negócio e seu desempenho operacional tem sido amplamente estudada. Várias sugestões podem ser encontradas na bibliografia acadêmica e na prática, enfatizando a importância de se estabelecer uma relação entre a eficácia na gestão e sua operação, traduzida em metas, objetivos e requisitos da organização (Broadbent e Weill, 1997; Carvalho e Laurindo, 2003a; 2003b; Kaplan e Norton, 1992; 1993; 1996; 2000; Neely e Austin, 2000; Prieto, 2006; Rockart, 1979; Tonchia, 2000).

Segundo Kennerly e Neely (2000), um sistema de medida de desempenho eficaz possui necessariamente as seguintes partes: "1 - métricas individuais que quantificam e eficiência e eficácia das ações; 2 - um conjunto de medidas que permita o acesso ao desempenho da organização como um todo; e 3 - uma estrutura que suporte e habilite a aquisição, ordenação, análise, interpretação e disseminação dos dados". O foco deste artigo está no ponto 2 de um sistema de medida de desempenho.

Os métodos atualmente mais referenciados são o Balanced Scorecard (BSC) (Kaplan e Norton, 1992), a Pirâmide de Desempenho - Performance Pyramide (Kerseens-van Drongelen, 2000) e o Prisma de Desempenho - Performance Prism (Kennerly e Neely, 2000), dentre os quais o $B S C$ tem se tornado o mais difundido nas empresas.

O objetivo deste artigo é indicar aspectos críticos e dificuldades da implementação do Balanced Scorecard e 
tentar entender quais são os conceitos e técnicas de gestão de negócio que suportam o processo de implementação desta metodologia.

Este artigo apresenta, nas seções 2 e 3, respectivamente, uma revisão da literatura sobre $B S C$ e sobre os fatores críticos para sua implementação. Na seção 4 , é apresentada a estratégia de pesquisa adotada, detalhando-se os aspectos metodológicos. Na seção 5, são apresentados os resultados da pesquisa empírica. Finalmente, a última seção apresenta as conclusões.

\section{Visão geral do balanced scorecard}

O Balanced Scorecard (BSC) desenvolvido por Kaplan e Norton em 1992 resultou das necessidades de captar toda a complexidade da performance na organização e tem sido ampla e crescentemente utilizado em empresas e organizações (Epstein e Manzoni, 1998).

Entre suas contribuições estão a composição e a visualização de medidas de performance que reflitam a estratégia de negócios da empresa. O BSC deve levar à criação de uma rede de indicadores de desempenho que deve atingir todos os níveis organizacionais, tornandose, assim, uma ferramenta para comunicar e promover o comprometimento geral com a estratégia da corporação (Kaplan e Norton, 1996; 2000).

Este método resume em um único documento, indicadores de performance em quatro perspectivas: financeira, clientes, processos internos e aprendizado e crescimento. (Kaplan e Norton, 1992; 1996).

Hauser e Katz (1998: p. 517) advertem: "toda métrica, independente da forma utilizada, irá afetar ações e decisões. Mas, evidentemente, escolher a certa é crítico para o sucesso". Neste sentido, cada perspectiva necessita de seu próprio e distinto conjunto de medidas de performance, as quais precisam ser específicas de acordo com as características e necessidades de cada empresa. Tais medidas precisam refletir e materializar a missão e estratégia da empresa (Kaplan e Norton, 1993).

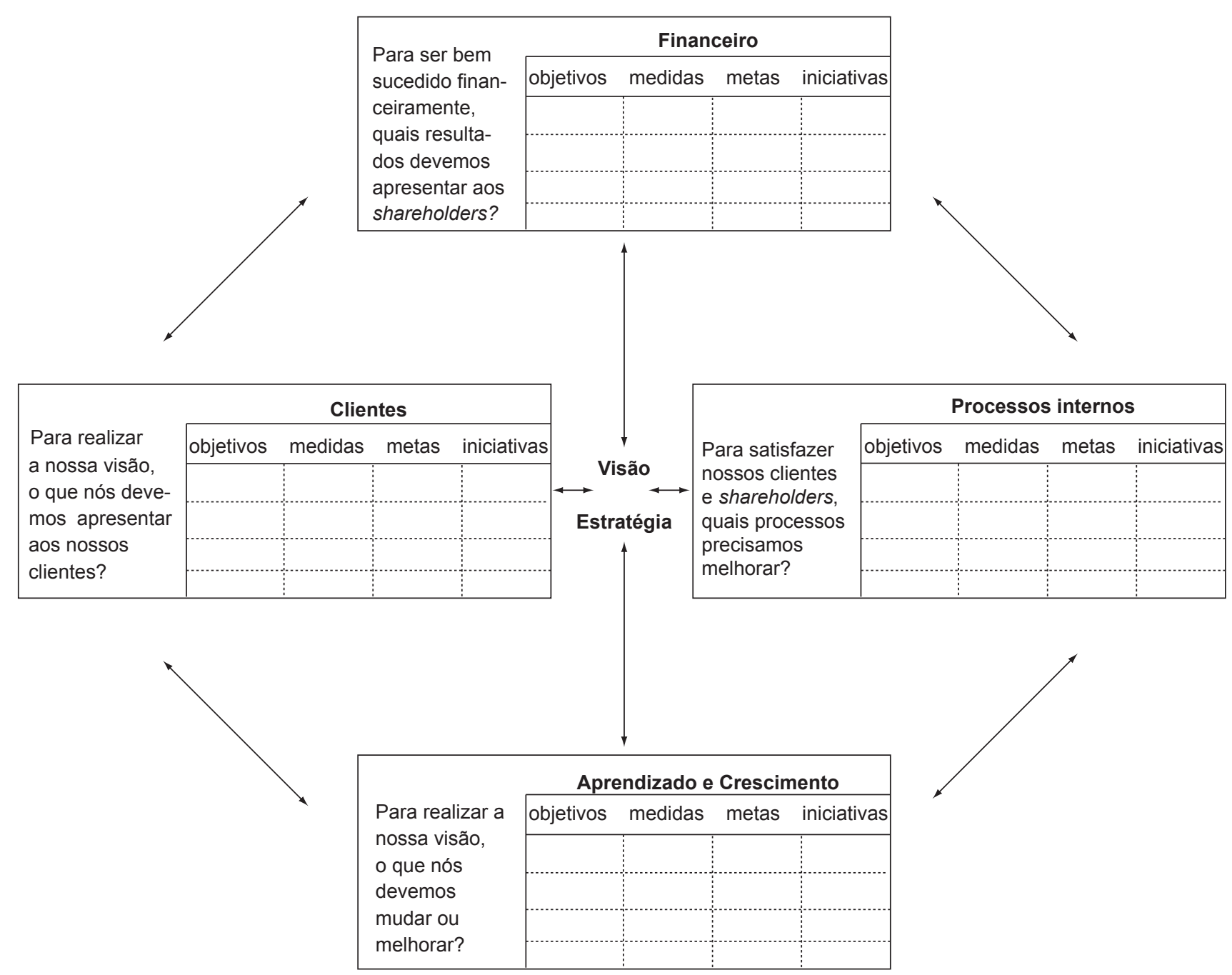

Figura 1. Traduzindo visão e estratégia: quatro perspectivas (adaptado de Kaplan e Norton, 1996). 
Para facilitar o entendimento da estratégia por toda a empresa, Kaplan e Norton (2000) propuseram o BSC. A Figura 1 ilustra o modelo do $B S C$. Cada perspectiva deve ter seu próprio conjunto de indicadores, formulados para viabilizar o cumprimento da estratégia e da visão da organização.

Perspectiva financeira: Monitora se a estratégia da empresa está contribuindo para a melhoria dos resultados financeiros. As metas financeiras se relacionam com rentabilidade, crescimento e valor para os acionistas.

Os objetivos e medidas financeiros desempenham um papel duplo: definem o desempenho financeiro esperado da estratégia e servem de meta principal para a definição dos objetivos e medidas das outras perspectivas do scorecard. Segundo Kaplan e Norton (2000), nesta perspectiva, as empresas trabalham com duas estratégias básicas: crescimento da receita e produtividade. A primeira irá refletir nas outras perspectivas, no sentido de gerar novas fontes de receita provenientes de novos mercados, novos produtos ou novos clientes ou na ampliação do relacionamento com os clientes existentes. Já a estratégia de produtividade irá refletir na busca da execução eficiente das atividades operacionais em apoio aos clientes atuais, podendo incluir, também, a redução de custos.

Perspectiva do cliente: Pressupõe definições quanto ao mercado e segmentos nos quais a organização deseja competir. A organização deverá traduzir em medidas específicas os fatores importantes para os clientes. A proposta é monitorar como a empresa entrega real valor ao cliente certo. Normalmente são definidos indicadores da satisfação e de resultados relacionados aos clientes: satisfação, retenção, captação e lucratividade.

Perspectiva dos processos internos: Os indicadores de perspectiva dos clientes e dos acionistas devem ser apoiados por processos internos. Nesta perspectiva as organizações identificam os processos críticos para a realização dos objetivos das duas perspectivas anteriores. Os processos devem criar as condições para que a organização ofereça propostas de valor ao cliente, capazes de atrair e reter clientes nos seus segmentos de atuação e, ao mesmo tempo, criando valor aos acionistas.

Perspectiva do aprendizado e do crescimento: Empresas com condição de serem cada vez melhores são empresas com capacidade de aprender. A capacitação da organização se dará por meio dos investimentos em novos equipamentos, em pesquisa e desenvolvimento de novos produtos, em sistemas e procedimentos e nos recursos humanos da empresa.

Visto de maneira integrada, o balanced scorecard traduz o conhecimento, habilidades e sistemas que os empregados precisarão (seu aprendizado e crescimento), para inovar e construir as capacidades estratégicas certas e eficientes (processos internos) que entregarão valor específico ao mercado (clientes), os quais, eventualmente, proporcionarão o aumento do valor ao acionista (financeiro).

Kaplan e Norton (1996) definem quatro processos gerenciais para implementação do $B S C$, conforme ilustra a Figura 2.

O processo de tradução da visão ajuda a construir um consenso em torno da visão e da estratégia da organização. Os autores advertem que devem ser evitadas declarações vagas como "o melhor da classe", pois o processo deve conduzir a definição das medidas de desempenho. O resultado deste processo é a tradução da estratégia em termos operacionais, ou seja, o desenho dos mapas estratégicos, $B S C s$.

O processo de comunicação e ligação - consiste em comunicar a estratégia no sentido vertical e horizontal da estrutura, ligando os objetivos departamentais aos indivi-

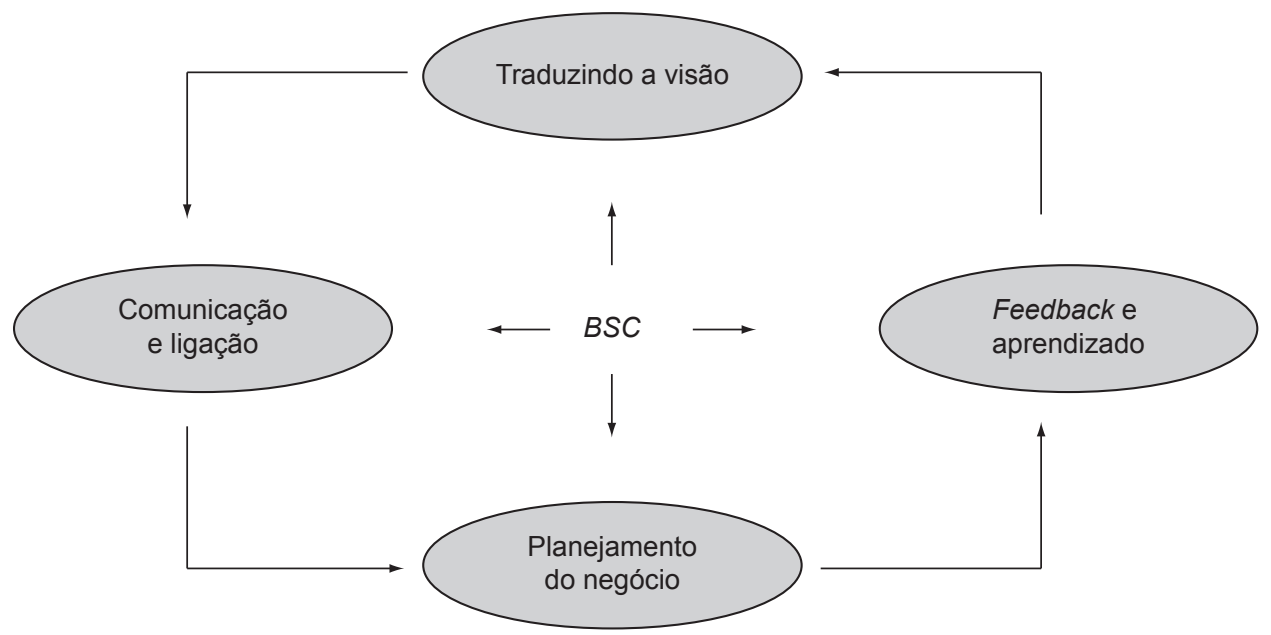

Figura 2. Gerenciando a Estratégia: quatro processos (adaptado de Kaplan e Norton, 1996). 
duais. Deve-se evitar que os objetivos individuais priorizem as metas de curto prazo, alinhando-os à estratégia da organização.

Este processo também consiste em identificar os processos-chave e desenvolver medidas de desempenho para estes processos.

O processo de planejamento do negócio consiste em alocar os recursos e definir as prioridades de acordo com as metas estratégicas.

O processo de feedback e aprendizado visa dar à organização a capacidade de aprendizado estratégico, ou seja, fazer da gestão estratégica um processo contínuo. Com o BSC, uma organização pode monitorar seus resultados de curto prazo nas quatro perspectivas, permitindo a modificação das estratégias em curso e refletindo o aprendizado organizacional.

Esses quatro processos refletem uma sequiência iterativa de ações. Para chegar a um sistema gerencialmente estabilizado, segundo o exemplo apresentado por Kaplan e Norton (1996), talvez seja preciso cerca de trinta meses, sendo que a organização pode percorrer esses quatro processos duas ou três vezes.

\section{Aspectos críticos na implementação do BSC}

De acordo com Kaplan e Norton (2000), Mercer (1999) e Schneiderman (1999), diversas empresas encontraram dificuldades na implementação do $B S C$, com o uso significativo de recursos humanos e financeiros, sem os resultados desejados. Kaplan (1999a) anuncia que "desenvolver e implementar um sistema de gerenciamento $B S C$ numa empresa não é uma iniciativa das mais simples". Para melhor entender a complexidade apontada por Kaplan, este artigo procura conjugar as principais dificuldades encontradas em termos globais, conforme a revisão de literatura, para criar e compor um guia para o desenvolvimento da pesquisa.

Um dos problemas mais comuns diz respeito ao envolvimento do gerente nível sênior, a partir do momento em que os executivos passam a delegar toda a estratégia do processo de implementação ao nível hierárquico intermediário. Kaplan (1999a) argumenta que estes gerentes intermediários podem não estar preparados para entender a estratégia nem o projeto do $B S C$ como um todo, não tendo nem a autoridade nem o conhecimento dos processos necessários para o sucesso da implementação. Estes autores também se referem ao comprometimento da gerência como fator tão importante como conhecimento e autoridade. Além disso, Kaplan (1999a) enfatiza que a posição da alta administração deve ser a de dividir funções e responsabilidades, de forma a evitar o isolamento durante a implementação do BSC. Kaplan (1999a, p. 3) diz, "O resultado final desta autoconfiança excessiva é que os processos de tomada de decisão, em níveis superiores, não mudam". Ele também destaca o ponto de vista de Norton a respeito da divisão de responsabilidades: "tirar a estratégia da mão de 10 e passar para 10 mil" (Kaplan, 1999a, p. 4). Para alcançar o propósito da empresa como um todo, Kaplan (1999a) sugere atribuir os scorecards da empresa gradualmente, distribuindo o esforço da implementação e também ajustando e refinando os objetivos e necessidades em todos os setores da empresa.

Segundo Kaplan (1999a), uma parte significativa do conjunto total de medidas do BSC não está disponível nos primeiros meses de implementação do processo. No entanto, isto não deve ser considerado um verdadeiro problema. Porém, em vez de se deter em detalhes sem fim em busca de um começo perfeito, as empresas deveriam começar pela utilização de medidas de $B S C$ e promover uma contínua melhoria para esta abordagem. Kaplan (1999b, p. 5) afirma: “o scorecard não é um evento único - é um processo de gerenciamento contínuo." Carvalho e Laurindo (2003b) alertam ainda que a implementação do $B S C$ anda junto com o gerenciamento por processos, se a empresa ainda não mapeou seus processos críticos, vai demorar para estabelecer as métricas nesta perspectiva.

Um outro importante relato de Kaplan (1999b) diz respeito a uma situação muito comum, quando consultores externos ficam responsáveis pela implementação do $B S C$. Em tal circunstância, há um alto risco de se produzir um sistema sofisticado, mas que não prioriza as necessidades de gestão do executivo sênior. Portanto, do ponto de vista de Kaplan (1999b, p. 5 e 6) "sistema e tecnologia são extremamente importantes...mas os sistemas e tecnologias aplicados devem vir após a gestão ter finalizado seu trabalho estratégico inicial, e que irão criar os objetivos, medidas, metas, iniciativas e scorecards interligados por toda empresa."

Hauser e Katz (1998) propõem que vantagens relacionadas com valores não devem ser baseadas apenas em planejamentos de longo prazo, mas também nos de curto prazo, de forma a manter a motivação individual. Por outro lado, Kaplan (1999) alerta para o risco de o resultado ser apenas uma simples adição de medidas não financeiras orientadas para a composição dos planos de compensação. Neste caso, cada medida específica de performance é melhorada, mas não a performance financeira total.

Ao implementar um sistema de medida de performance, existe o risco de se produzirem valores incompatíveis com o uso de modelos (comparativos) sem a devida caracterização/personalização. Kaplan e Norton (1993) alertam para o fato e demonstram que as diferentes situações de mercados, segmentos de negócios, estratégias de produtos e ambientes competitivos demandam diferentes scorecards. 
Kaplan e Norton (1993) ressaltam a importância de manter equilibradas as quatro principais perspectivas do $B S C$ : "Este conjunto equilibrado de medidas demonstra não só as negociações que gerentes já efetivaram através de medidas de performance, como os incentiva a alcançar suas metas no futuro sem fazer negociações entre fatores de sucesso".

Hauser e Katz (1998) ressaltam uma importante questão durante a implementação de um sistema de medida de performance: evitar que a baixa capacidade de empregados e gerentes em diversificar riscos afete a escolha de projetos em função de métricas não claras que permitam a escolha pelo menor risco, comprometendo as probabilidades de resultados de longo prazo. Desta forma, isto pode criar um custo de risco que pode levar a empresa a se desviar de seus objetivos a longo prazo. Hauser e Katz (1998) também afirmam que a utilização de valores hard to control podem criar uma certa resistência à sua utilização pelos gerentes do nível intermediário. Para evitar este problema, os autores sugerem escolher valores que possam ser implementados a curto prazo, mas fortemente ligados aos objetivos a longo prazo da empresa, numa correlação altamente estratégica. Hauser e Katz (1998) preferem estes valores adequados, estrategicamente alinhados, mesmo quando não são precisos, do que valores de alta precisão e sem importância para uma abordagem produtiva. Além disso, há o risco de se perderem os objetivos organizacionais e de se manter a velha cultura baseada em antigos valores.

Conforme Kaplan (2000), discussões inconsistentes, assim como informação e comunicação improdutivas, são os maiores impedimentos para a total compreensão dos objetivos e da estratégia da empresa por parte dos funcionários. Kaplan (2000) reconhece que onze em cada quinze seguidores bem sucedidos do $B S C$ utilizam sistemas formais para avaliação de conhecimentos dos funcionários. Embora de vital importância para o sucesso do BSC, Kaplan e Norton (1993) demonstraram que o processo de comunicação pode ser impactado pelo risco de vir à tona informação estratégica a investidores externos ou competidores, mas é importante se organizar com dados confidenciais de forma a se evitarem impactos negativos na comunicação e na implementação de objetivos internos.

Um importante fundamento de Kaplan e Norton (1996) diz respeito à falta de definição de uma estratégia comum e sua transição para objetivos claros. O simples conhecimento das metas corporativas não é suficiente para mudar o comportamento das pessoas; é necessário incentivar a aliança dos objetivos e medidas, associando o nível individual às metas de longo prazo da empresa. Desta forma, um scorecard corporativo deverá envolver a definição de objetivos comuns e temas a serem adotados por todas as unidades de negócios, promovendo uma sinergia que irá produzir um valor maior para a empresa. Além disso, cada modelo (comparativo) em larga escala se refletirá em metas locais (Kaplan e Norton, 1999).

Um outro erro comum apresentado por Kaplan e Norton (1999) consiste em considerar o BSC simplesmente como um projeto para desenvolver métricas, em vez de um projeto de mudanças estratégicas que incluem cada detalhe da empresa. A consequiência deste erro é que pode levar a resultados inexpressivos. Bons resultados exigem gerenciamento de tempo e ênfase em prioridades bem definidas para que se possa implementar uma estratégia vista de cima para baixo. Por último, a equipe encarregada da implementação do $B S C$ é de extrema importância. As pessoas envolvidas na condução do projeto $B S C$ devem compor uma equipe estrategicamente posicionada, multifuncional e integrada, de forma a analisar em detalhes a estratégia e os valores da empresa como um todo. Deve-se evitar que a equipe seja constituída apenas por um grupo de especialistas em suas funções.

\section{Aspectos metodológicos}

A proposta deste artigo é discutir os fatores críticos de sucesso na implementação do $B S C$, bem como o processo de mudança e as técnicas envolvidas nos bastidores da implementação, tendo como objeto central o confronto entre a perspectiva da empresa de consultoria para implementação do $B S C$ e da empresa que adota o método.

A abordagem metodológica utilizada pode ser descrita conforme a Figura 3. Em uma primeira fase da pesquisa foi elaborada uma estrutura teórica (framework), extraída da literatura apresentada nas seções 2 e 3 deste artigo. Esta estrutura foi submetida a empresas de consultoria focadas na implementação de $B S C$ para identificar e listar os fatores críticos para o sucesso das implementações. Posteriormente, um estudo de caso foi realizado com o objetivo de analisar os mesmos aspectos sob o ponto de vista das empresas que adotam a metodologia, as empresas clientes. A empresa selecionada é representativa para a pesquisa por ter adotado o $B S C$ em quatro das suas uni-

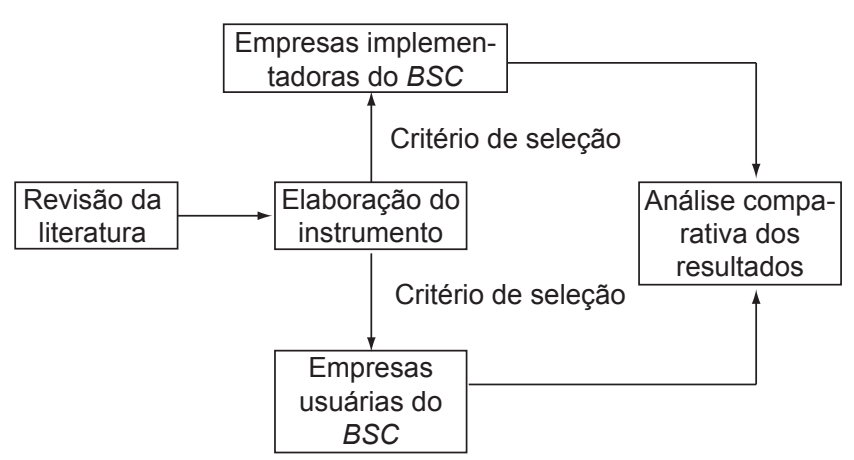

Figura 3. Abordagem metodológica. 
dades com diferentes graus de sucesso na implementação. A abordagem de estudo de caso foi adotada por ser mais adequada para investigar um fenômeno contemporâneo, sem limites claros e definições (Yin, 2005). Conforme sugere Bonoma (1985), foram utilizadas diversas fontes de informação além das entrevistas, tais como o estudo de documentos e dados dos sistemas de métricas utilizados.

O critério para seleção dos casos de empresas que implementam o BSC foi baseado em número de casos implementados em empresas nacionais e a disponibilidade no fornecimento das informações para a pesquisa. Baseadas neste critério, foram selecionadas quatro empresas de consultoria. Além da informação qualitativa obtida nas entrevistas, alguns dados quantitativos foram reunidos a partir de 14 projetos de implementação de $B S C$ desenvolvidos pelas quatro empresas estudadas cujos documentos também foram analisados para reforçar os estudos da pesquisa (Eisenhardt, 1989).

Os critérios para a seleção do caso de empresa que utiliza o $B S C$ foram: adoção do $B S C$ a mais de um ano e não ser cliente das empresas de consultoria de implementação do $B S C$ selecionadas para o estudo. Os principais aspectos estudados na empresa selecionada foram: 1) como as situações críticas apontadas pelas firmas de consultoria foram conduzidas durante a implementação do $B S C$ (ver Tabela 4); 2) se de fato elas ocorreram; 3 ) se elas poderiam ter sido evitadas; e 4) assim como o impacto para o sucesso da implementação.

\subsection{Instrumento de pesquisa e roteiro}

Para a elaboração do instrumento de pesquisa foram listados os fatores críticos apresentados nas seções teóricas deste artigo, resultando em 26 questões principais, como mostra a Tabela 1 . O instrumento de pesquisa incluía estas questões, distribuídas aleatoriamente numa folha de registro, no qual deveriam ser colocados os comentários sobre os projetos de implementação do $B S C$.

A Tabela 2 apresenta a planilha organizada para receber os dados das entrevistas.

\section{Resultados da pesquisa de campo}

Nesta seção, são apresentados os resultados da pesquisa empírica tanto sob a perspectiva das empresas de consultoria como das empresas usuárias do $B S C$. No final da seção é feita a análise comparativa dos resultados.

\subsection{A perspectiva das empresas de consul- toria para implementação do BSC}

Quatro empresas de consultoria brasileiras foram pesquisadas, utilizando as técnicas de estudo de casos descrita na seção anterior. Destas empresas coletou-se um conjunto de 14 implementações que foram submetidas à avaliação de situações críticas, de acordo com a Tabela 3.

\subsubsection{Resultados da pesquisa}

Depois de preenchidas as folhas de registro, as entrevistas foram executadas com as respectivas empresas de consultoria e finalmente os dados pesquisados foram resumidos de acordo com a Tabela 4.

Dois assuntos foram apontados em $93 \%$ dos projetos como os mais comuns e mais atuantes: comprometimento da alta administração e discussões não claras e infreqüentes. As principais justificativas e abordagens apresentadas para diminuir o risco dessas situações foram a criação de um processo formal de gestão de mudança organizacional e a melhoria do processo de comunicação (Kaplan, 1999a). Essas duas situações podem ser classificadas como não relacionadas de forma específica ao método $B S C$. A terceira situação mais comum foram quatro perspectivas não balanceadas, relatadas em

Tabela 1. Aspectos críticos agrupados e submetidos para entrevista.

\begin{tabular}{|c|c|}
\hline Itens & Aspectos críticos \\
\hline 1 & Comprometimento da alta administração \\
\hline 2 & Ação isolada da alta administração \\
\hline 3 & Não dividir papéis e responsabilidades \\
\hline 4 & $B S C$ como evento único e não como processo contínuo \\
\hline 5 & $B S C$ como um projeto de sistema \\
\hline 6 & Discussões não claras e infrequentes \\
\hline 7 & Quatro perspectivas não balaceadas \\
\hline 8 & Uso do $B S C$ apenas para remuneração variável \\
\hline 9 & $\begin{array}{l}\text { Uso do } B S C \text { como um projeto de métrica e não de } \\
\text { estratégia }\end{array}$ \\
\hline 10 & Falta de senso de urgência \\
\hline 11 & Não ter um time focado durante a implementação \\
\hline 12 & Não ter uma visão estratégica comum \\
\hline 13 & Não conseguir traduzir a estratégia \\
\hline 14 & Não estar apto a desdobrar para unidades de negócio \\
\hline 15 & $\begin{array}{l}\text { Comunicação e Treinamento não efetivo nas diversas } \\
\text { fases }\end{array}$ \\
\hline 16 & Falta de alinhamento entre métricas e objetivos \\
\hline 17 & $\begin{array}{l}\text { Não estabelecer um alinhamento entre estratégia métri- } \\
\text { ca com o orçamento }\end{array}$ \\
\hline 18 & $\begin{array}{l}\text { Problemas de comunicação de dados estratégicos } \\
\text { sensíveis }\end{array}$ \\
\hline 19 & Implementar BSC como "template" \\
\hline 20 & $\begin{array}{l}\text { Atraso nas recompensas - Recompensar somente no } \\
\text { longo prazo }\end{array}$ \\
\hline 21 & $\begin{array}{l}\text { Usar métricas ousadas - incorrer no custo de evitar } \\
\text { risco }\end{array}$ \\
\hline 22 & Usar métricas difíceis de controlar \\
\hline 23 & Perder os objetivos de vista \\
\hline 24 & Escolher métricas precisamente incorretas \\
\hline 25 & Assumir que empregados não tem outras opções \\
\hline 26 & Não quebrar paradigmas \\
\hline
\end{tabular}


Tabela 2. Planilha organizada para receber dados das entrevistas.

\begin{tabular}{cccccc}
\hline $\begin{array}{c}\text { Número de casos sobre entrevista } \\
\text { Item }\end{array}$ & Aspecto critico & Frequência & Resultado & Mitigação & Outras observações \\
\hline 1 & & & & & \\
2 & $\Downarrow$ & $\Downarrow$ & $\Downarrow$ & $\Downarrow$ & $\Downarrow$ \\
$\Downarrow$ & & & & & \\
26 & & & & & \\
\hline
\end{tabular}

Tabela 3. Característica da amostra.

\begin{tabular}{cclcc}
\hline Consultoria & Projetos analisados & \multicolumn{1}{c}{ Indústria } & $\begin{array}{c}\text { Tempo médio de } \\
\text { implementação }\end{array}$ & $\begin{array}{c}\text { Número médio de } \\
\text { pessoas no projeto }\end{array}$ \\
\hline 1 & 10 & $\begin{array}{l}\text { Construção, utilities, produtos de } \\
\text { consumo e softwares }\end{array}$ & 1 ano e meio & 15 \\
2 & 1 & Produtos de consumo & 6 meses & 7 \\
3 & 1 & Utilities & 6 meses \\
4 & 2 & Serviços financeiros & 1 ano & 9 \\
\hline
\end{tabular}

Tabela 4. Resultados da pesquisa.

\begin{tabular}{clcc}
\hline Itens & \multicolumn{1}{c}{ Aspectos críticos } & Ocorrências & Percentual de ocorrência \\
\hline 1 & Compromentimento da alta administração & 13 & 93 \\
6 & Discussões não claras e infrequentes & 13 & 93 \\
7 & Quatro perspectivas não balaceadas & 11 & 79 \\
4 & $B S C$ como evento único e não como processo contínuo & 9 & 64 \\
3 & Não dividir papéis e responsabilidades & 8 & 57 \\
5 & $B S C$ como um projeto de sistema & 8 & 57 \\
2 & Ação isolada da alta administração & 3 & 21 \\
13 & Não conseguir traduzir a estratégia & 3 & 21 \\
19 & Implementar $B S C$ como "template" & 3 & 21 \\
\hline
\end{tabular}

$79 \%$ dos casos. Essa situação pode ser classificada como relativa à técnica do $B S C$. As atitudes de mitigação desta situação se concentram em parte na comunicação, envolvimento da alta administração e informação gerencial e parte na experiência da empresa em manter, equilibrar e coordenar as quatro perspectivas $B S C$ de acordo com a estratégia da empresa. Foi também observado que os indicadores financeiros foram priorizados em comparação com outras perspectivas do $B S C$. A respectiva moderação sugerida para minimizar esta questão foi acompanhar a relação de causa e efeito no mapa estratégico.

O BSC como único evento e não como processo contínuo: tal questão teve destaque em $64 \%$ das citações. Esta situação acontece quando a empresa não apresenta uma política de melhoria contínua e enfatiza apenas o sistema de medida de performance do $B S C$ devido a pressões para que se termine o projeto. Além disso, empresas de consultoria vão precisar transferir knowhow para conduzir as operações de BSC após o final do projeto de implementação. Comunicação e informação durante o projeto de implementação poderiam minimizar tal situação.

Não dividir papéis e responsabilidades representa 57\% das citações, cuja causa é não haver uma equipe designada para isto. Isto poderia ser contornado por meio de uma distribuição formal de funções e responsabilidades destacando as necessidades do projeto e atribuindo objetivos gradualmente ao pessoal qualificado. Outras causas foram relacionadas aos baixos cálculos e ao baixo envolvimento gerencial em relação ao projeto, o que está fortemente associado com a necessidade de utilização de suporte técnico tais como uma gestão de projeto.

Em 57\% dos casos surgiu o tópico BSC como um projeto de sistema. Uma observação coletada durante a entrevista exemplifica esta questão: “... a maior parte das empresas vê o $B S C$ como um projeto tecnológico baseado no fato da grande incidência de projetos liderados pelos Departamentos de Sistemas". Esta moderação adequada foi baseada na criação de comitês formais de gestão superior para liderar o projeto e para delegar po- 
deres a outras áreas da empresa na implementação do $B S C$.

Por fim, três questões menos relevantes $(21 \%$ das ocorrências) foram citadas: ação isolada da alta administração; não conseguir traduzir a estratégia; e implementar BSC como template. Uma interessante observação foi a seguinte: “...é muito comum encontrar um defensor solitário do conceito de $B S C$, uma vez que não se tem conhecimento e maturidade sobre o $B S C$ e gestão de desempenho".

\subsection{A perspectiva das empresas clientes}

A empresa usuária do BSC selecionada como objeto deste estudo é a terceira maior instituição financeira privada do Brasil.

A implementação do $B S C$ foi sugerida ao Presidente e à diretoria por assessores internos que tomaram conhecimento da ferramenta no meio acadêmico. A alta administração aprovou a implementação e nomeou um comitê que conduziu todo o processo de introdução do $B S C$ na empresa.

\subsubsection{O processo de implementação}

A implementação do BSC teve início no final de 1999, nas quatro unidades de negócio do banco - varejo, atacado, seguros e gestão do patrimônio - e foi estruturado dentro de um ano. A corporação definiu a visão de futuro que foi desmembrada em objetivos estratégicos e em fatores críticos de sucesso (FCS), de acordo com as 4 perspectivas propostas por Kaplan e Norton, com base nas diretrizes estratégicas das unidades de negócio. BSCs alinhados, a corporação passou, então, a explicar a metodologia aos seus quase 30.000 funcionários.

O plano de comunicação estabelecido para a implementação do $B S C$ utilizou uma linguagem simples, criativa e de fácil memorização. A própria ferramenta foi introduzida na empresa com um nome diferente do original - PDG - Painel de Gestão - para facilitar a comunicação.

Os navegadores catarinenses da família Schürmann foram contratados para dar palestras fazendo uma analogia da sua experiência com o papel dos executivos na concretização da estratégia. O acionista e presidente da corporação participou diretamente da divulgação aos executivos e gravou um vídeo, que foi exibido a todos os funcionários, quando da apresentação da proposta a eles, pelos executivos.

O processo de implementação evoluiu com intensidade diferente em cada uma das unidades de negócios e algumas delas encontraram maior dificuldade em familiarizarse com a ferramenta. Uma unidade que se destacou pela forma de implementação do $B S C$ foi a unidade de seguros. O presidente da unidade participou ativamente de todo o processo. Foi criado um símbolo para o programa, um leão, batizado de LEO - Liderança, Espírito Empreendedor e
Organização -, pensando nos atributos que seriam necessários para alcançar as metas propostas. Nesta unidade, a visão de futuro que vigorou de 2000 a 2002 foi definida como 3-3-3: ser, em três anos, um dos três maiores grupos seguradores em três aspectos: participação no mercado, satisfação dos clientes e lucro líquido. A empresa obteve bons resultados quanto à satisfação dos clientes.

De 2003 a 2006 uma nova visão de futuro foi delineada. Desta vez o processo recebeu o nome de Copa do Milhão, devido a viagens à Copa da Alemanha que serão proporcionadas às equipes que conseguirem atingir as metas. A visão de futuro é "ser a primeira companhia do mercado em pessoa física até 2006 , consolidando a liderança em pessoa jurídica". O objetivo é vender 1.000 .000 de apólices em cada um dos produtos foco, que são os principais produtos da seguradora. É importante ressaltar que a definição de metas de curto prazo tem o objetivo de manter a motivação individual e, dentro da proposta do $B S C$, é válida desde que se traduza na melhoria da performance financeira total.

\subsubsection{Principais resultados}

\section{Aprendizado sobre o mapeamento da estratégia:}

- Cada unidade tinha a sua forma de implementação da estratégia e o foco era nos resultados financeiros. O BSC criou uma padronização em torno das quatro perspectivas, incluindo indicadores para os ativos intangíveis;

- O fato de estarem sendo utilizados indicadores comuns a todas as unidades (indicadores de tendência) faz com que se eleve o nível de prestação de contas, levando a uma análise conjunta dos resultados e ao benchmarking;

- A importância atribuída aos indicadores de tendência vai aumentando com o aprendizado, à medida que o executivo consegue visualizar que determinado desempenho financeiro tem uma relação direta com tais indicadores;

- A implementação gera um processo de aprendizado em torno da própria estratégia e do uso de ferramentas de gestão; e

- O mapeamento da estratégia deixa claro quais são os fatores críticos de sucesso para o negócio e serve para definir a prioridade dos investimentos.

\section{Acompanhamento dos resultados}

O Comitê de Planejamento Executivo, formado pelo acionista presidente da corporação e pelos presidentes das unidades de negócios, analisa mensalmente os indicadores financeiros e trimestralmente todas as perspectivas. No total são de 20 a 30 indicadores estratégicos. 


\section{Perspectiva resultado}

Qual deverá ser o resultado do meu trabalho ao final do período?

Diz respeito aos projetos e desafios cujo resultado implica numa redução de custo, acréscimo de receita ou maior eficácia para minha área/negócio.

Caso a área possua o seu Painel de Gestão, estas atividades devem estar em linha com os Fatores Críticos de Sucesso da perspectiva financeira do mapa estratégico.

Ex.: 1) Projeto de redução de despesas administrativas da área em 5\%; e

2) Desenvolver uma proposta de acompanhamento da retenção de estagiários.

\section{Perspectiva clientes}

Quais necessidades de meus clientes devo atender a fim de melhorar o relacionamento e aumentar sua satisfação?

Diz respeito ao cumprimento e entrega de atividades pré-definidas/acordadas que impactam diretamente em clientes internos/externos. Implica também numa postura pró-ativa quanto ao atendimento das suas necessidades.

Caso a área possua o seu Painel de Gestão, estas atividades devem estar em linha com os Fatores Críticos de Sucesso da perspectiva clientes do mapa estratégico.

Ex.: 1) Disponibilizar o modelo de avaliação de empresas requisitado pela Controladoria dentro do prazo estipulado; e

2) Realizar palestras para minhas áreas clientes a fim de elevar o entendimento dos principais produtos da área.

Quais processos internos tenho de realizar ou aprimorar?

Diz respeito àquelas atividades cujo cumprimento ou aprimoramento é essencial para aumentar a eficiência operacional da área.

Caso a área possua o seu Painel de Gestão, estas atividades devem estar em linha com os Fatores Críticos de Sucesso da perspectiva interna do mapa estratégico.

Ex.: 1) Unificar a base de dados relativa a treinamento institucional; e

2) Disponibilizar diariamente as principais notícias do mercado financeiro no portal corporativo.

\section{Perspectiva aprendizado e crescimento}

O que devo fazer para constantemente aprender novos conceitos e aprofundar meus conhecimentos de forma a melhor contribuir para os desafios da minha área e crescer profissionalmente?

Diz respeito ao seu plano de desenvolvimento, educação e treinamento que deve ser efetuado no período que possa melhorar seu desempenho.

Caso a área possua o seu Painel de Gestão, estas atividades devem estar em linha com os Fatores Críticos de Sucesso da perspectiva aprendizado e crescimento do mapa estratégico.

Ex.: 1) Aumentar a capacitação em mercado financeiro; e

2) Aprofundar meus conhecimentos relativos a Marketing de Relacionamento.

Figura 4. Roteiro para configuração das 4 perspectivas do contrato de gestão na empresa $A$.

No dia a dia, cada diretoria da unidade faz a gestão dos seus indicadores.

\section{Contrato de gestão}

No esforço de desdobrar a estratégia para todos os níveis organizacionais, cada um dos funcionários da Empresa A tem hoje um contrato de gestão (Figura 4) elaborado nos mesmos moldes do BSC. O contrato de gestão é constituído por objetivos individuais desmembrados nas 4 perspectivas. Tais objetivos estão relacionados aos objetivos estratégicos da área em que o funcionário trabalha, que por sua vez estão relacionados à estratégia da corporação.

Neste aspecto, vêm sendo realizadas as primeiras ações para atrelar a remuneração variável aos indicadores do $B S C$. No entanto, este aspecto é considerado crítico pela empresa. Por um lado, existe a necessidade e a expectativa da recompensa pelos objetivos alcançados, e por outro lado a dificuldade na sua mensuração. Questões como a diversificação dos objetivos, a qualidade da sua elaboração, a coexistência de objetivos qualitativos e quantitativos, dificultam a comparação entre eles e a definição de critérios justos de recompensa.

\section{Mudanças no sistema de gestão}

Algumas mudanças estão sendo implementadas para atender aos fatores críticos de sucesso definidos no mapa estratégico, como por exemplo:

- A Unidade de Seguros, na perspectiva dos clientes, definiu como um dos fatores críticos de sucesso, conhecer bem os nossos clientes e suas necessidades. Para atender a esta necessidade foram criadas duas áreas, a de pessoa física e a de pessoa jurídica. Também foi criada uma base de dados única, englobando os produtos (seguro auto, vida e casa) para integrar todas as informações disponíveis de um mesmo cliente;

- Fazer uso das melhores práticas de gestão, foi definido como fator crítico de sucesso na perspectiva de aprendizado e crescimento. O resultado foi a introdução de um 
portal de gestão, que armazena todas as informações necessárias para a gestão dos recursos humanos;

- Na perspectiva interna foi definido como fator crítico oferecer produtos e serviços competitivos em todos os canais de venda e conquistar fatia de mercado para os produtos definidos. Foram definidos produtos específicos para serem vendidos pelos agentes de seguro e uma competição, chamada rally, foi introduzida para alavancar as vendas de outros seguros, que não os de automóveis; e

- Remunerar e reconhecer em linha com os objetivos estratégicos da corporação é um fator crítico de sucesso da perspectiva de aprendizado e crescimento. A empresa está estudando uma forma de introduzir uma recompensa variável atrelada aos objetivos estratégicos de cada área.

\subsection{Análise comparativa dos resultados das pesquisas}

Algumas situações críticas apontadas na seção 5.1 pelas firmas de consultoria foram evidenciadas durante o processo de implementação na empresa A, conforme relato abaixo:

Falta de comprometimento da alta administração e discussões não claras e pouco freqüentes foram os dois principais aspectos apontados pelos implementadores como impactando os demais. A pesquisa conduzida na empresa A confirma a importância destes aspectos para assegurar o sucesso na implementação do BSC. Como relatado no caso, a unidade de seguros avançou mais rapidamente em todo o processo de introdução do $B S C$, assimilou mais rapidamente o conceito da ferramenta e a utiliza no processo decisório. O presidente da unidade participou ativamente de todo o processo de implementação e tem um time de trabalho especificamente engajado na gestão do BSC. Semanalmente, o presidente reúne-se com este time e com os principais executivos para acompanhar os resultados dos indicadores.

Por outro lado, na unidade de Atacado, o processo de comunicação do $B S C$ precisou ser revisado porque o $B S C$ era discutido apenas uma vez por mês, na reunião de planejamento.

Uma das soluções encontradas foi permitir que os executivos da unidade tivessem acesso ao software que gerencia os resultados da unidade como um todo.

$\mathrm{O}$ terceiro aspecto apontado foram as quatro perspectivas não balanceadas. A introdução do $B S C$ na empresa A é recente. Depois de três anos, a empresa fez o seu segundo mapa estratégico. $\mathrm{O}$ estágio atual é de consolidação da aplicação da ferramenta e também de aprendizado sobre a importância das medidas que monitoram aspectos intangíveis do negócio. A tendência de dar maior importância aos indicadores financeiros é relevante, até mesmo pela natureza do negócio em questão.

O BSC como único evento e não como processo contínuo: Ficou evidenciado que o BSC requer algumas ações complementares. A empresa vem trabalhando em um sistema de recompensas atrelado aos objetivos estratégicos e no mapeamento das competências individuais.

Não dividir papéis e responsabilidades: $O$ processo de implementação na empresa foi uma iniciativa interna, conduzida por um time formado especificamente para este propósito. Os indicadores e metas foram definidos em conjunto com os principais executivos de cada área. O time encarregado da implementação decidiu que não seria prioridade que os executivos se envolvessem com a sistemática do $B S C$, mas com a definição das métricas.

\section{Conclusões}

Os resultados da pesquisa de campo indicaram que alguns fatores críticos para o sucesso na implementação do $B S C$ não são praticados, entre eles destaca-se a falta de comprometimento da alta administração (93\%). Nos casos analisados verificou-se que é freqüente que a condução do $B S C$ seja feita pela média gerência, com foco em resultados e métricas de curto prazo, que geram um viés no método que deve conduzir a ações estratégicas e não táticas.

Observa-se que os processos de implementação de sucesso estudados possuíam uma estrutura formal e processos de comunicação bem definidos, atrelados a um forte alinhamento estratégico, desdobrados em processos críticos também bem definidos, para as quatro perspectivas estratégicas, pois sem uma estratégia que permeie todos os níveis hierárquicos da empresa, as chances de sucesso no alcance dos objetivos organizacionais são mínimas.

Algumas situações críticas apontadas tanto pelas firmas de consultoria como pela empresa usuária do $B S C$ : discussões não claras e pouco freqüentes; quatro perspectivas não balanceadas; $B S C$ como único evento e não como processo contínuo; e não dividir papéis e responsabilidades.

Além destes fatores, outros aspectos correlatos que devem ser investigados em uma agenda futura de pesquisa são: a qualidade da estratégia, a estrutura de comunicação e de mudança de cultura, o processo de premiação e distribuição dos ganhos, adequação dos indicadores que monitoram os ativos intangíveis. 


\section{Referências Bibliográficas}

BONOMA, T. V. Case research in marketing: opportunities, problems and process. Journal of Marketing Research. v. 22, n. 2, p. 199-208, may, 1985.

BROADBENT, M.; WEIL, P. Management by maxim: how business and it managers can create it infrastructures. Sloan Management Review, v. 38, n. 3, p. 77-92, Spring, 1997.

CARVALHO, M. M.; LAURINDO, F. J. B. Estratégias para a competitividade, São Paulo: Futura, 2003a.

Linking strategy with a network of performance indicators: a brazilian research centre. International Journal of Business Performance Management, v. 5, n. 4, p. 285-301, 2003b.

EISENHARDT, K. M. Building theories from case study research. Academy of Management Review. v. 14, n. 4, p. 532 - 550, 1989.

EPSTEIN, M.; MANZONI, J. F. Implementing corporate strategy: from tableaux de bord to balanced scorecards. European Management Journal, v. 16, n. 2, p. 190-203, 1998.

HAUSER, J.; KATZ, G. Metrics: you are what you measure! European Management Journal. v. 16, n. 5, p. 517-528, 1998.

KAPLAN, R. S. Can bad things happen to good scorecards? Harvard Business School Press: Balanced Scorecard: insight, experience e ideas for strategyfocused organization report. Article B9911D, 1999a.

Can bad things happen to good scorecards? Part II of implementation pitfalls. Harvard Business School Press: Balanced Scorecard: Insight, experience e ideas for strategy-focused organization report. Article B9909D, 1999b.

Communication and education to make strategy everyone's job. Harvard Business School Press, 2000.

KAPLAN, R. S.; NORTON, D. P. The balanced scorecard: measures that drive performance. Harvard Business Review, v. 70, n. 1, p. 71-79, jan./feb. 1992.

Putting the balanced scorecard to work. Harvard Business Review. v. 71, n. 5, p. 134-142, sep./oct. 1993.
Using the balanced scorecard as a strategic management system. Harvard Business Review. v. 74, n. 1, p. 75-85, jan./feb. 1996.

Building a Strategy-Focused Organization. Harvard Business School Press, 1999.

Having Trouble With Your Strategy? Then map it. Harvard Business Review, v. 78, n. 5, p. 167-176, sep./oct. 2000.

KENNERLY, M.; NEELY, A. Performance measurement framework - a review. In: Performance measurement - past, present and future. UK: Andy Neely, Centre for Business Performance, Cranfield School of Management, Cranfield University, Cranfield, Bedfordshire, 2000.

KERSEENS-VAN DRONGELEN, I. C. Systematic design of performance measurement systems. In: Performance Measurement - past, present and future. UK: Andy Neely, Centre for Business Performance, Cranfield School of Management, Cranfield University, Cranfield, Bedfordshire, 2000.

MERCER, W. M. Balanced scorecards determine employees rewards. News Release. v. 5. New York: Willian M. Mercer, Inc., apr. 1999.

NEELY,A.;AUSTIN, R. Measuring operations performance: past, present and future. In: PERFORMANCE MEASUREMENT, 2000, CONFERENCE. Proceedings... Cambridge, p. 419-426, 2000.

PRIETO, V.C. Análise de modelos de alinhamento estratégico interno. 2006. 145p. Dissertação (Mestrado em Engenharia de Produção) - Escola Politécnica da Universidade de São Paulo. São Paulo, 2006.

ROCKART, J. F. Chief executives define their own data needs. Harvard Business Review. v. 57, n. 2, p. 81-92, mar./apr. 1979.

SCHNEIDERMAN, A. Why balanced scorecards fail. Journal of strategic performance measurement. Special Edition 6, p. 6-11. jan. 1999.

TONCHIA, S. Linking performance measurement system to strategic and organizational choices. International Journal of Business Performance. v. 2, n. 1/2/3, p. 15-29, 2000.

YIN, Robert. Estudo de Caso: Planejamento e Métodos. Porto Alegre: Bookman, 2005. 


\title{
CRITICAL FACTORS IN BALANCED SCORECARD IMPLEMENTATION
}

\begin{abstract}
A growing body of studies focuses on the relevance of aligning business strategies and its operational performance. Several reports in the literature emphasize the importance of linking effectiveness to the goals, objectives and requirements of organizations to allow for the ongoing management of operational performance. Today, one of the most popular methods for supporting an effective performance measurement system is the Balanced Scorecard (BSC), which has been widely and increasingly used in many different kinds of businesses and organizations. This paper discusses the critical factors determining the successful implementation of the BSC, based on a comparative analysis of a BSC consulting firm and a client company implementing the BSC. The methodological approach adopted here was a multiple case study.
\end{abstract}

Keywords: balanced scorecard (BSC), strategic alignment, strategic implementation, performance measurement systems. 